\title{
A fresh look at keV sterile neutrino dark matter from frozen-in scalars
}

\author{
Adisorn Adulpravitchai $^{a}$ and Michael A. Schmidt ${ }^{b}$ \\ ${ }^{a}$ Department of Physics, Faculty of Science, Chulalongkorn University, \\ Bangkok 10330, Thailand \\ ${ }^{b}$ ARC Centre of Excellence for Particle Physics at the Terascale, \\ School of Physics, The University of Sydney, \\ NSW 2006, Australia \\ E-mail: adisorn.adulpravitchai@gmail.com, \\ m.schmidt@physics.usyd.edu.au
}

ABSTRACT: Sterile neutrinos with a mass of a few $\mathrm{keV}$ can serve as cosmological warm dark matter. We study the production of $\mathrm{keV}$ sterile neutrinos in the early universe from the decay of a frozen-in scalar. Previous studies focused on heavy frozen-in scalars with masses above the Higgs mass leading to a hot spectrum for sterile neutrinos with masses below $8-10 \mathrm{keV}$. Motivated by the recent hints for an X-ray line at $3.55 \mathrm{keV}$, we extend the analysis to lighter frozen-in scalars, which allow for a cooler spectrum. Below the electroweak phase transition, several qualitatively new channels start contributing. The most important ones are annihilation into electroweak vector bosons, particularly $W$-bosons as well as Higgs decay into pairs of frozen-in scalars when kinematically allowed.

Keywords: Beyond Standard Model, Cosmology of Theories beyond the SM, Neutrino Physics

ARXIV EPRINT: 1409.4330 


\section{Contents}

1 Introduction 1

2 Model 2

3 Dark matter production 4

$\begin{array}{lll}4 & \text { Free-streaming horizon } & 7\end{array}$

5 UV completion $\quad 11$

6 Conclusion 12

$\begin{array}{ll}\text { A Cross sections and decay widths } & 13\end{array}$

$\begin{array}{ll}\text { B Thermal averages } & 14\end{array}$

$\begin{array}{ll}\text { C Effective degree of freedom } & 14\end{array}$

\section{Introduction}

Sterile Neutrinos are a well motivated minimal extension of the Standard Model (SM) of particle physics [1]. In particular sterile neutrinos with a keV-scale mass and tiny mixing with active neutrinos are able to explain the cosmological abundance of DM. The keVscale of the sterile neutrinos can be explained in numerous ways, for instance, see [2-8]. In contrast to standard cold dark matter (CDM), they generally are warmer and have a larger free-streaming horizon. Thus they can be candidates for warm dark matter (WDM). As WDM candidates, they suppress structure at small scales and are able to solve the missing satellite problem [9-11]. Additionally they might explain the velocities of pulsars [12, 13].

As keV sterile neutrinos generally mix with the active neutrinos, they are produced non-thermally via oscillations $[14,15]$, which is called the Dodelson-Widrow (DW) mechanism [16] in the case of keV sterile neutrinos. The DW mechanism is excluded by observation [17, 18]. However the bounds can be avoided, if there is a large enough primordial lepton asymmetry and sterile neutrinos are produced via resonant oscillations, the Shi-Fuller mechanism [19]. Further examples for production mechanisms are (a) the production from the decay of a scalar field which is in thermal equilibrium with the thermal plasma $[12,20,21]$, (b) the production from inflaton decays [22, 23], (c) the thermal production by new gauge interactions and subsequent dilution by production of entropy [24, 25] and (d) the production via the decay of a frozen-in scalar [26].

We will focus on the production of $\mathrm{keV}$ sterile neutrinos from the decay of a frozen-in scalar [26]. In this mechanism the sterile neutrino couples to a real scalar field and both 
couple extremely weakly to the thermal bath of SM particles. In addition to the activesterile neutrino mixing, there is the coupling of the Higgs with the new scalar. The scalar is produced via freeze-in [27] and subsequently decays to the $\mathrm{keV}$ sterile neutrino [26]. The discussion in ref. [26] restricts the frozen-in scalar to be heavier than the SM Higgs. This however implies that $\mathrm{keV}$ sterile neutrinos with masses below $8-10 \mathrm{keV}$ form hot dark matter (HDM), erase too much structure at small scales, and thus are excluded.

This work is motivated by the recent hint for an X-ray line at approximately $3.55 \mathrm{keV}$ by two analyses of the XMM-Newton data [28, 29], which might be explained by sterile neutrino dark matter (DM) with a mass of approximately $7.1 \mathrm{keV}[21,30-37]$. Other explanations for the line are given in refs. [38-75]. Irrespective whether the hints for the X-ray line persist $[76,77]$ or disappear [78-80], keV sterile neutrinos are excellent WDM candidates, including $\mathrm{keV}$ sterile neutrinos with a mass below $10 \mathrm{keV}$. Thus we are studying the production of a $\mathrm{keV}$ sterile neutrino from a frozen-in scalar without restricting the frozen-in scalar mass to be larger than the Higgs mass.

Above the electroweak (EW) phase transition, the only process contributing to the production of the frozen-in scalar is Higgs annihilation. ${ }^{1}$ Frozen-in scalars lighter than the critical temperature of the EW phase transition lead to several new processes contributing to their production and consequently the DM abundance: SM fermion-antifermion annihilation, weak gauge boson annihilation, and Higgs decay to two frozen-in scalars if kinematically allowed. There is also the direct decay of the SM Higgs boson to a pair of $\mathrm{keV}$ sterile neutrinos. However it is negligible in the region of parameter space we study. The most important processes are Higgs annihilation and annihilation into EW vector bosons above $m_{h} / 2$ and Higgs decay below $m_{h} / 2$, when it becomes kinematically allowed. The fermion-antifermion annihilation is generally sub-dominant. Neutrino masses can be generated in different ways. We will firstly use an effective operator approach for neutrino mass and give one possible UV completion using the type-II seesaw mechanism [82-87].

The paper is organised as follows. In section 2, we introduce the model. In section 3 , the dark matter production is explained. In section 4, we discuss the free-streaming horizon of the dark matter in order to determine whether the $\mathrm{keV}$ sterile neutrino constitutes HDM, WDM, or CDM. In section 5 we give one possible UV completion of the effective operator for neutrino mass. Finally we conclude in section 6 . Technical details are collected in the appendices. Cross section and decay widths can be found in appendix A. Their thermal averages are given in appendix B. A short summary of the treatment of effective degrees of freedom is given in appendix $\mathrm{C}$.

\section{Model}

Apart from the SM particle fermion content, we introduce one light SM singlet fermion $N$ and one scalar singlet $\phi$. For simplicity we introduce a discrete $Z_{4}$ version of lepton

\footnotetext{
${ }^{1}$ If a singlet condensate forms during inflation, there is an additional contribution to the production from the decay of the condensate [81].
} 
number $^{2}$ with the following transformation properties of the fields

$$
L \rightarrow i L \quad E^{c} \rightarrow-i E^{c} \quad N \rightarrow-i N \quad H \rightarrow H \quad \phi \rightarrow-\phi .
$$

The most general Yukawa interactions in the lepton sector and the dimension 6 operator generating neutrino mass are given by

$$
-\mathcal{L}=y_{E} L H E^{C}+y_{L N} L H N+\frac{1}{2} y_{N} \phi N^{2}+\frac{y_{\nu}}{\Lambda^{2}} L L H H \phi+\text { h.c. } .
$$

After all scalars obtain a vacuum expectation value (vev)

$$
H=\left(\begin{array}{c}
G^{+} \\
v+\frac{1}{\sqrt{2}}\left(h+i G^{0}\right)
\end{array}\right) \quad \phi=v_{\phi}+\sigma,
$$

with the Goldstone bosons $G^{ \pm}, G^{0}$, the active neutrino mass matrix is given by

$$
m_{\nu}=y_{\nu} \frac{v_{\phi} v^{2}}{\Lambda^{2}}-\frac{y_{L N} y_{L N}^{T}}{y_{N}} \frac{v^{2}}{v_{\phi}}
$$

We are interested in the region of parameter space with electroweak scale vevs $v \sim v_{\phi}$ and small couplings $y_{L N}^{2} \ll y_{\nu} y_{N} v_{\phi}^{2} / \Lambda^{2}$, which results in a negligibly small seesaw contribution to the light neutrino mass.

The most general scalar potential is given by the scalar potential

$$
V=-\mu_{H}^{2} H^{\dagger} H+\frac{\lambda_{H}}{6}\left(H^{\dagger} H\right)^{2}+\frac{\lambda_{H \phi}}{2} H^{\dagger} H \phi^{2}-\frac{1}{2} \mu_{\phi}^{2} \phi^{2}+\frac{\lambda_{\phi}}{24} \phi^{4}
$$

and the minimisation of the potential yields the vevs $v=\sqrt{3 \mu_{H}^{2} / \lambda_{H}}$ and $v_{\phi}=\sqrt{6 \mu_{\phi}^{2} / \lambda_{\phi}}$ in the limit of a small Higgs portal coupling $\lambda_{H \phi}$. At leading order there is no mixing between the different scalar fields. The scalar masses at leading order are given by

$$
m_{h}^{2}=2 \mu_{H}^{2}=\frac{2 \lambda_{H}}{3} v^{2} \quad \text { and } \quad m_{\sigma}^{2}=2 \mu_{\phi}^{2}=\frac{\lambda_{\phi}}{3} v_{\phi}^{2} .
$$

The only relevant mixing for the production via freeze-in is the mixing between the Higgs doublet and the singlet $\phi$, which is given by

$$
\tan (2 \gamma)=\frac{\lambda_{H \phi}}{\sqrt{2}} \frac{v v_{\phi}}{\mu_{H}^{2}-\mu_{\phi}^{2}}=\frac{3 \sqrt{2} \lambda_{H \phi} v v_{\phi}}{2 \lambda_{H} v^{2}-\lambda_{\phi} v_{\phi}^{2}} .
$$

\footnotetext{
${ }^{2}$ The breaking of the discrete symmetry $Z_{4}$ could lead to the formation of domain walls [88], which alter the history of the universe. There are several different ways to avoid this problem. See for example refs. [89-93]. The mechanism even works without imposing a $Z_{4}$ symmetry, but the following additional terms are introduced

$$
\mathcal{L}_{\not / 4}=\left(\frac{1}{2} m_{N}^{\prime} N^{2}+\text { h.c. }\right)+\rho_{1} \phi+\rho_{2} \phi^{3}+\rho_{3} H^{\dagger} H \phi .
$$

Obviously the tadpole term of $\phi$ can be removed by shifting $\phi$. If the explicit mass term of $N$ is of the correct size and the coupling $\rho_{3} H^{\dagger} H \phi$ is sufficiently small, the production of a keV sterile neutrino from a frozen-in scalar similarly works without the $Z_{4}$ symmetry.
} 


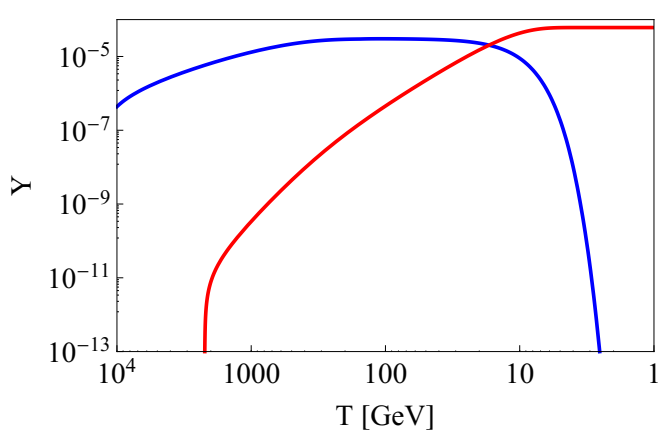

(a) $m_{\sigma}=500 \mathrm{GeV}$.

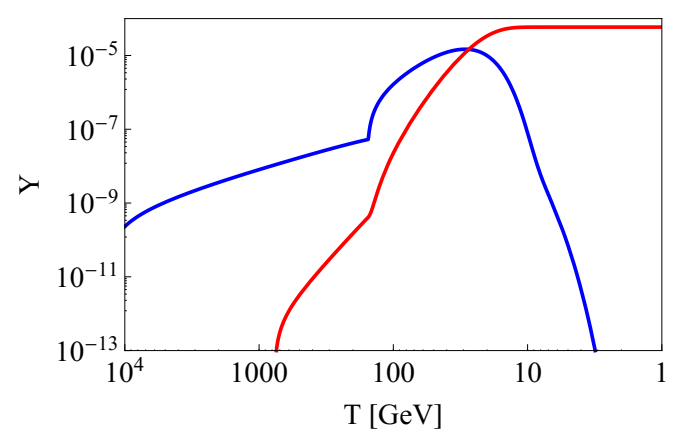

(b) $m_{\sigma}=60 \mathrm{GeV}$.

Figure 1. Typical evolution of the abundances of the scalar $\sigma$ (shown in blue) and the keV sterile neutrino $N$ (shown in red). The sterile neutrino mass is fixed to $m_{N}=7.1 \mathrm{keV}$ and $\lambda_{\phi}=0.5$.

The relevant couplings for the production of dark matter via freeze-in are the two Higgs portal interactions

$$
\Delta V=\lambda_{H \phi} \frac{h^{2} \sigma^{2}}{4}+\sqrt{2} \lambda_{H \phi} v \frac{h \sigma^{2}}{2} .
$$

The decay of the sterile neutrino to an active neutrino and a photon is determined by the mixing with active neutrino flavour $\alpha$ via the small mixing angle

$$
\theta_{\alpha} \approx \frac{y_{L N, \alpha} v}{y_{N} v_{\phi}}
$$

According to $[28,29]$, if we want to explain the $3.55 \mathrm{keV}$ line from the decay of the sterile neutrino DM, the active-sterile mixing is constrained to be $\sum_{\alpha} \sin ^{2}\left(2 \theta_{\alpha}\right) \simeq 7 \times 10^{-11}$.

\section{Dark matter production}

We consider the sterile neutrino production in the early universe. If the keV sterile neutrino gets into thermal equilibrium with SM particles, it will be overproduced and will overclose the universe after freeze-out. Therefore usually a keV sterile neutrino is very weakly coupled to the thermal bath and it is assumed that the initial abundance is zero. We concentrate on the freeze-in mechanism as main production mechanism, which has been first discussed in [26]. In the freeze-in mechanism, keV sterile neutrinos are produced in two steps. First a feebly-coupled scalar field, $\sigma$, is produced via a tiny Higgs portal coupling, $\lambda_{H \phi}$, which has to be small enough such that $\sigma$ is always out of thermal equilibrium. This scalar subsequently decays into keV sterile neutrinos. The authors of ref. [26] consider scalars with a mass heavier than the Higgs mass. In this region of parameter space the main process is Higgs annihilation $(h h \leftrightarrow \sigma \sigma)$. See figure 1a for a typical evolution of the abundances of the $\mathrm{keV}$ sterile neutrino (red) and the scalar $\sigma$ (blue) for a frozen-in scalar with a mass larger than the mass of the Higgs. As it can be seen in [26] and we explain in more detail in the next section, it turns out that heavy scalars lead to larger free-streaming scales for light keV sterile neutrinos, like a $7.1 \mathrm{keV}$ sterile neutrino. Thus it is interesting to consider scalars lighter than the Higgs and consequently below the EW phase transition. In addition to Higgs annihilation, there are the following additional processes: 
- annihilation of vector bosons: $V V \leftrightarrow \sigma \sigma$ with $V=W, Z$,

- annihilation of SM fermions: $\bar{f} f \leftrightarrow \sigma \sigma$

- Higgs decay to pairs of the scalar $\sigma$ as well as pairs of keV sterile neutrinos directly.

A typical evolution for a frozen-in scalar with mass $m_{\sigma}=60 \mathrm{GeV}$ is shown in figure $1 \mathrm{~b}$. Note that the production of the $\mathrm{keV}$ sterile neutrino is dominated by Higgs decay and very weakly depends on the physics above the EW phase transition. The abundances $Y_{\sigma, N} \equiv n_{\sigma, N} / s$ normalised to the entropy density $s$ are described by the following Boltzmann equations:

$$
\frac{d Y_{\sigma}}{d T}=\frac{d Y_{\sigma}^{\mathrm{A}}}{d T}+\frac{d Y_{\sigma}^{\mathrm{D}}}{d T}+\frac{d Y_{\sigma}^{\mathrm{HD}}}{d T} \quad \frac{d Y_{N}}{d T}=\frac{d Y_{N}^{\mathrm{D}}}{d T}+\frac{d Y_{N}^{\mathrm{HD}}}{d T}
$$

where the superscripts A, D, and HD denote annihilation, decay and Higgs decay terms, respectively. Before the SM particles get out of thermal equilibrium, the different terms are given by

$$
\begin{aligned}
\frac{d Y_{\sigma}^{\mathrm{A}}}{d T} & =\sqrt{\frac{\pi}{45 G_{N}}} \sqrt{g_{*}(T)} \sum_{i=h, W, Z, t, b, c, \tau}\langle\sigma v(\sigma \sigma \rightarrow i i)\rangle\left(Y_{\sigma}(T)^{2}-Y_{\sigma}^{e q}(T)^{2}\right) \\
\frac{d Y_{\sigma}^{\mathrm{D}}}{d T} & =-\frac{1}{2} \frac{d Y_{N}^{\mathrm{D}}}{d T} \\
\frac{d Y_{N}^{\mathrm{D}}}{d T} & =-\sqrt{\frac{45}{\pi^{3} G_{N}}} \frac{1}{T^{3}} \frac{1}{\sqrt{g_{\mathrm{eff}}(T)}}\langle\Gamma(\sigma \rightarrow N N)\rangle\left(Y_{\sigma}(T)-\left(\frac{Y_{N}(T)}{Y_{N}^{e q}(T)}\right)^{2} Y_{\sigma}^{e q}(T)\right) \\
\frac{d Y_{\sigma}^{\mathrm{HD}}}{d T} & =-\sqrt{\frac{45}{\pi^{3} G_{N}}} \frac{1}{T^{3}} \frac{1}{\sqrt{g_{\mathrm{eff}}(T)}}\langle\Gamma(H \rightarrow \sigma \sigma)\rangle\left(1-\left(\frac{Y_{\sigma}(T)}{Y_{\sigma}^{e q}(T)}\right)^{2}\right) Y_{h}^{e q}(T) \\
\frac{d Y_{N}^{\mathrm{HD}}}{d T} & =-\sqrt{\frac{45}{\pi^{3} G_{N}}} \frac{1}{T^{3}} \frac{1}{\sqrt{g_{\mathrm{eff}}(T)}}\langle\Gamma(H \rightarrow N N)\rangle\left(1-\left(\frac{Y_{N}(T)}{Y_{N}^{e q}(T)}\right)^{2}\right) Y_{h}^{e q}(T) .
\end{aligned}
$$

$Y_{X}^{e q}(T)=n_{X}^{e q}(T) / s(T)$ denotes the equilibrium abundance, $G_{N}$ Newtons constant, $g_{\text {eff }}(T)$ the effective degrees of freedom at temperature $T$, and $g_{*}(T)$ is defined in the usual way. The precise definitions of the thermally averaged cross sections and decay widths as well as $g_{\text {eff }}$ and $g_{*}$ are collected in the appendices.

We show the different contributions for six different benchmark points. We fix the keV sterile neutrino mass to $m_{N}=7.1 \mathrm{keV}$ and the scalar self-coupling $\lambda_{\phi}=0.5$, which fixes the vev of the scalar singlet $\sigma$. We choose the Higgs portal coupling $\lambda_{H \phi}$ such that the observed DM abundance of $\Omega_{D M}=0.1199 \pm 0.0027$ [94] is obtained at $2 \sigma$. We also include the contribution from the DW mechanism using the approximate formula [95]

$$
\Omega_{N, D W} h^{2} \approx 0.2 \times \frac{\sum_{\alpha} \sin ^{2} \theta_{\alpha}}{3 \times 10^{-9}}\left(\frac{m_{N}}{3 \mathrm{keV}}\right)^{1.8}
$$

fixing $\sum_{\alpha} \sin ^{2}\left(2 \theta_{\alpha}\right)=7 \times 10^{-11}$. All parameter choices are collected in table 1 . The scalar vev, $v_{\phi}$, varies between $73 \mathrm{GeV}$ and $1.3 \mathrm{TeV}$ for our chosen benchmark points. The evolution of the abundances and the different contributions are shown in figure 2 for the six benchmark points. 


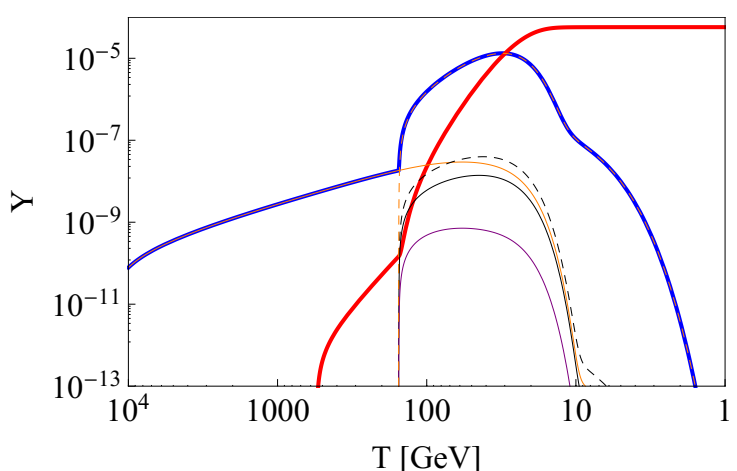

(a) $m_{\sigma}=30 \mathrm{GeV}$.

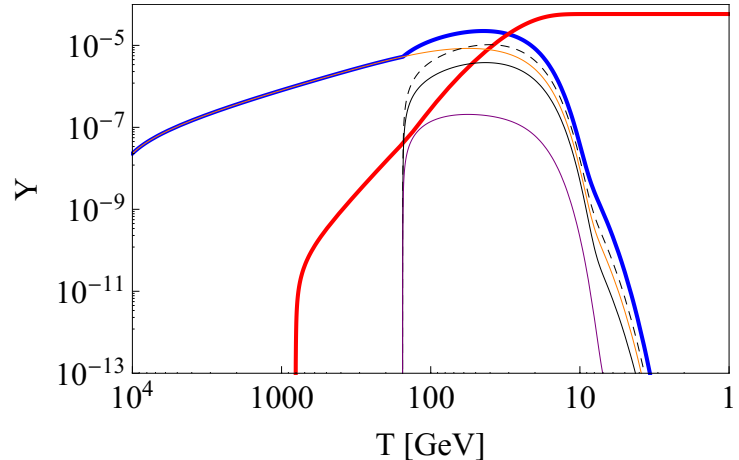

(c) $m_{\sigma}=65 \mathrm{GeV}$.

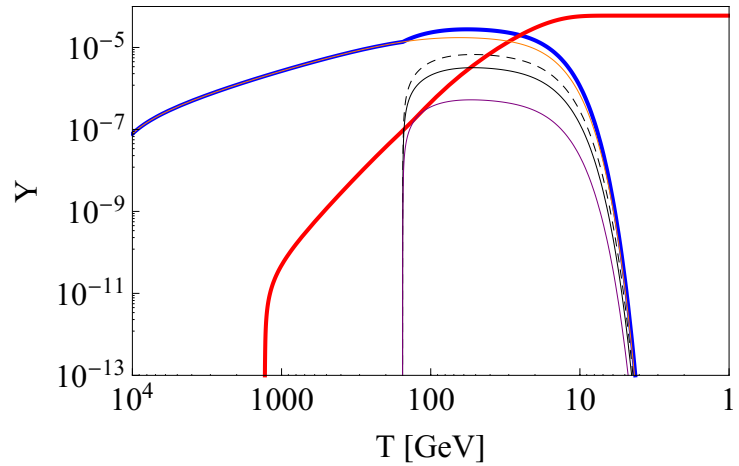

(e) $m_{\sigma}=170 \mathrm{GeV}$.

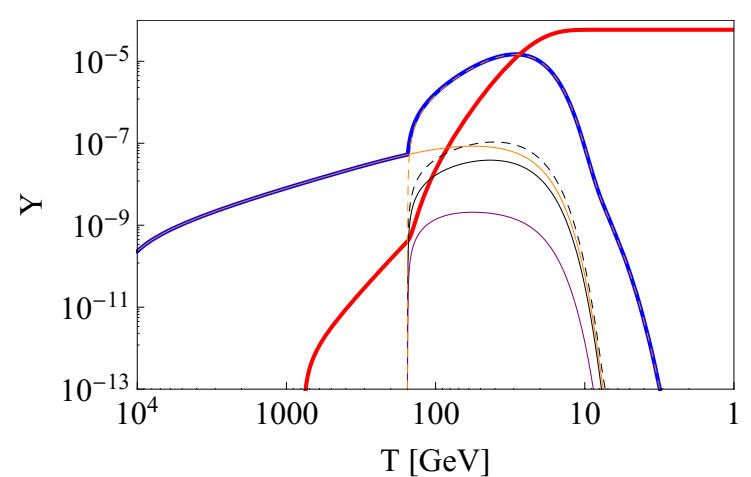

(b) $m_{\sigma}=60 \mathrm{GeV}$.

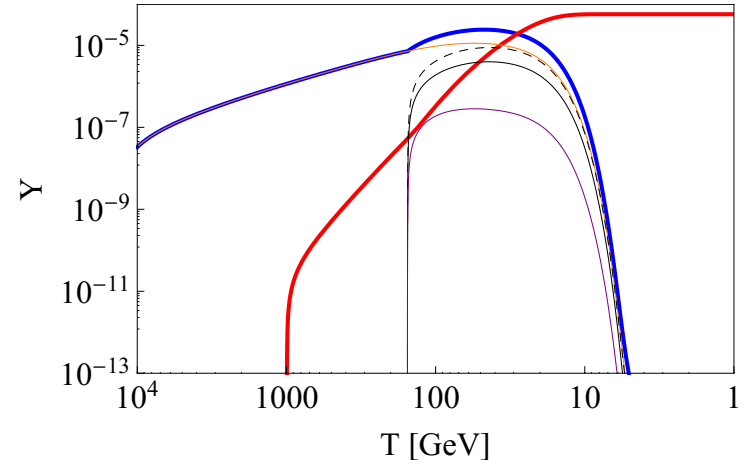

(d) $m_{\sigma}=100 \mathrm{GeV}$.

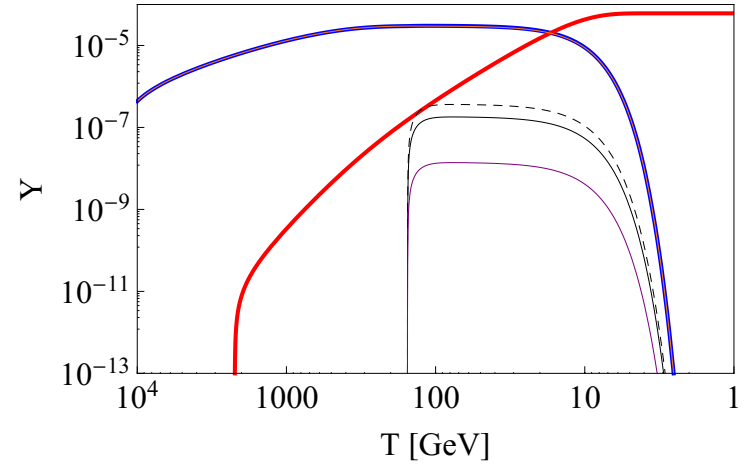

(f) $m_{\sigma}=500 \mathrm{GeV}$.

Figure 2. DM production for six benchmark points. The thick red (blue) line indicates the abundance of the $\mathrm{keV}$ sterile neutrino (scalar $\sigma$ ). The orange solid (dashed) line describes the contributions of Higgs annihilation (decay) to the abundance of $\sigma$. The annihilation of $Z$-bosons ( $W$-bosons) is described by the black solid (dashed) line. The contribution of $t \bar{t}$ annihilations are shown in purple. 


\begin{tabular}{|lcccccc|}
\hline$m_{\sigma}[\mathrm{GeV}]$ & 30 & 60 & 65 & 100 & 170 & 500 \\
$\lambda_{H \phi}\left[10^{-9}\right]$ & 3.65 & 6.26 & 62.6 & 75.0 & 116 & 274 \\
$\Omega_{N} h^{2}$ & 0.1174 & 0.1199 & 0.1188 & 0.1175 & 0.1218 & 0.1239 \\
$T_{\text {in }}[\mathrm{GeV}]$ & 17.8 & 16.3 & 18.1 & 16.4 & 13.8 & 8.77 \\
$r_{F S}[\mathrm{Mpc}]$ & 0.018 & 0.035 & 0.034 & 0.054 & 0.10 & 0.40 \\
& & & & & & \\
\hline
\end{tabular}

Table 1. Benchmark points. We fix $\lambda_{\phi}=0.5$ and the keV sterile neutrino mass $m_{N}=7.1 \mathrm{keV} . T_{\text {in }}$ denotes the temperature when $80 \%$ of the keV sterile neutrino DM are produced and $r_{F S}$ denotes the free-streaming horizon scale, which is discussed in section 4 .

Similarly to figure 1, the blue solid line shows the evolution of the abundance of the scalar $\sigma$ and the red solid line, the evolution of the abundance of the keV sterile neutrino. The orange solid (dashed) line describes the contributions of Higgs annihilation (decay) to the abundance of $\sigma$. The annihilation of $Z$-bosons ( $W$-bosons) is described by the black solid (dashed) line. The contribution of $t \bar{t}$ annihilations are shown in purple. The annihilation into light fermions is generally sub-dominant compared to $t \bar{t}$ annihilation. (See e.g. $[42,96])$. Note that the contribution from the Higgs decay to pairs of sterile neutrinos is negligible for our chosen parameters. We do not show its contribution in the plots. Higgs annihilation is the only process above the EW phase transition, because the other processes are proportional to the EW vev and are absent above the EW phase transition. In our numerical analysis we simply set the corresponding annihilation cross section to zero above the EW phase transition. A proper treatment requires the inclusion of thermal corrections to properly treat the temperature dependence during the EW phase transition. Below the EW phase transition we have to distinguish between frozen-in scalars with masses $m_{\sigma}>m_{h} / 2$ and $m_{\sigma}<m_{h} / 2$ : for $m_{\sigma}>m_{h} / 2$ there is a sizeable contribution from EW gauge boson annihilations, particularly annihilation of $W$-bosons, besides Higgs annihilation, as it can be seen in the plots for $m_{\sigma}=65,100 \mathrm{GeV}$, but for $m_{\sigma}<m_{h} / 2$, the most important process is Higgs decay (See the plots for $m_{\sigma}=30 \mathrm{GeV}$ and $m_{\sigma}=60 \mathrm{GeV}$.). As the coupling $\lambda_{H \phi}$ is extremely small, the contribution to the invisible decay width of the Higgs is negligibly small.

\section{Free-streaming horizon}

The free-streaming horizon can be used as the indicator whether the keV sterile neutrinos are HDM, WDM, or CDM. It is the comoving mean distance which a collision-less gravitationally unbound particle travels

$$
r_{\mathrm{FS}}=\int_{t_{\mathrm{in}}}^{t_{0}} \frac{\langle v(t)\rangle}{a(t)} d t
$$

where $t_{0}$ denotes time today, $t_{\text {in }}$ is its production time, $\langle v(t)\rangle$ its mean velocity at time $t$, and $a(t)$ the scale factor at time $t$. 
In our discussion we will neglect the DW contribution and concentrate on the main production mechanism via decays of frozen-in scalars. The DW contribution generally leads to a larger free-streaming horizon scale, since it is hotter than the contribution from the frozen-in scalar. For the parameters that we have chosen, the contribution of the DW mechanism represents less than $5 \%$ of the cosmological abundance, which is consistent with all known constraints. A detailed study of the free-streaming horizon scale is beyond the scope of this paper.

Guided by our numerical results in figure 2, we estimate the production time $t_{\mathrm{in}}{ }^{3}$ of the $\mathrm{keV}$ sterile neutrinos to be the time, when $80 \%$ have been produced. ${ }^{4}$

We follow the discussion of the free-streaming horizon in [26]. See ref. [97] for an earlier discussion applied to gravitino DM. We assume an instantaneous transition between the relativistic and the non-relativistic regimes of the $\mathrm{keV}$ sterile neutrino, i.e. $\langle v(t)\rangle \simeq 1$ for $t\left\langle t_{\mathrm{nr}}\right.$ and $\langle v(t)\rangle \simeq \frac{\langle p(t)\rangle}{m_{N}}$ for $t \geq t_{\mathrm{nr}}$, where $t_{\mathrm{nr}}$ is the time when the particle becomes non-relativistic, that is $\left\langle p\left(t_{\mathrm{nr}}\right)\right\rangle=m_{N}$.

Our numerical results in figure 2 show that the frozen-in scalar with masses $m_{\sigma} \gtrsim$ $30 \mathrm{GeV}$ mostly decays when it is non-relativistic. If the frozen-in scalar is lighter, a significant fraction will already decay when they are relativistic. We will focus on frozen-in scalar with masses $m_{\sigma} \gtrsim 30 \mathrm{GeV}$ and thus can safely assume that it decays non-relativistically. The rest of the discussion follows ref. [26]. The distribution of the DM produced from a non-relativistic parent $\sigma$ is given by [98-101]

$$
f(p, t)=\frac{\beta}{p / T_{\mathrm{DM}}} e^{-p^{2} / T_{\mathrm{DM}}^{2}}
$$

where $\beta$ is a normalisation factor and $T_{\mathrm{DM}}=p_{c m} a\left(t_{d}\right) / a(t)$ is the DM temperature. Using the DM momentum in the centre-of-mass frame,

$$
p_{c m}=\frac{\sqrt{m_{\sigma}^{2}-m_{N}^{2}}}{2} \simeq \frac{m_{\sigma}}{2}
$$

and the decay time $t_{d} \equiv t_{\text {in }}$,

$$
T_{\mathrm{DM}}(t) \simeq \frac{m_{\sigma}}{2} \frac{a\left(t_{\mathrm{in}}\right)}{a(t)}
$$

The average momentum $\langle p(t)\rangle$ can be calculated to be

$$
\langle p(t)\rangle=\frac{\int d^{3} p p f(p, t)}{\int d^{3} p f(p, t)}=\frac{\int_{0}^{\infty} d p p^{2} e^{-p^{2} / T_{\mathrm{DM}}^{2}}}{\int_{0}^{\infty} d p p e^{-p^{2} / T_{\mathrm{DM}}^{2}}}=\frac{\sqrt{\pi}}{2} T_{\mathrm{DM}} .
$$

\footnotetext{
${ }^{3}$ The relation between the production time $t_{\text {in }}$ and the production temperature $T_{\text {in }}$ is given by $t_{\text {in }} \simeq$ $\left(1.5 g_{*, S}^{-1 / 4}\right)^{2}\left(\frac{\mathrm{MeV}}{T_{\text {in }}}\right)^{2}$ sec.

${ }^{4}$ This differs from the approach of ref. [26], where the production time was estimated as $t_{\text {in }}=t_{\text {prod }, \sigma}+\tau$ where $t_{\text {prod, } \sigma}(\tau)$ is the production (decay) time of $\sigma$. In ref. [26] the production time $t_{\text {prod, } \sigma}$ was estimated to be the time when $\sigma$ becomes non-relativistic which is correct above the Higgs mass, but breaks down for light scalars $\sigma$. Our definition agrees with the one used in ref. [26] for $m_{\sigma} \gtrsim 100 \mathrm{GeV}$.
} 


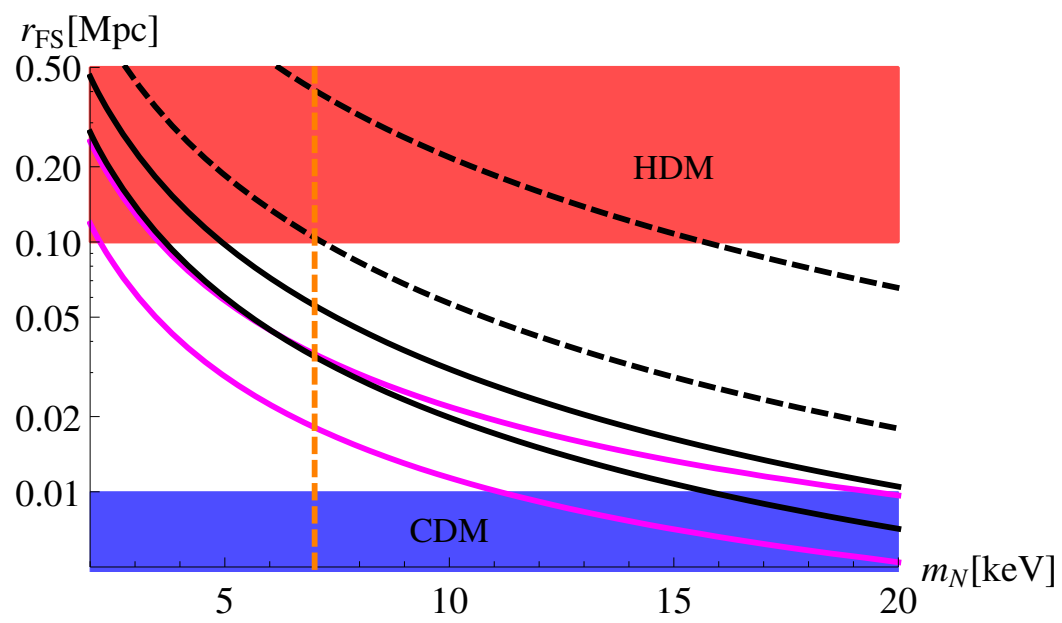

Figure 3. Free-streaming horizon vs. keV sterile neutrino mass. The regions of HDM and CDM are marked red and blue, respectively. The black and magenta lines show the free-streaming scale $r_{F S}$ for different masses of the frozen-in scalar. We assume a vanishing active-sterile mixing, $\theta=0$. From the lowest to the highest line the masses of the frozen-in scalar are $m_{\sigma}=30,60 \mathrm{GeV}$ (magenta solid lines), $m_{\sigma}=65,100 \mathrm{GeV}$ (black solid lines) and $m_{\sigma}=170,500 \mathrm{GeV}$ (black dashed lines). The vertical orange dashed line indicates $m_{N}=7.1 \mathrm{keV}$.

In the radiation dominated era, the scale factor $a \propto t^{1 / 2}$. Thus from eq. (4.4) and $\left\langle p\left(t_{\mathrm{nr}}\right)\right\rangle=m_{N}$, the time when $N$ becomes non-relativistic is given by

$$
t_{\mathrm{nr}}=\frac{\pi}{16} \frac{m_{\sigma}^{2}}{m_{N}^{2}} t_{\mathrm{in}}
$$

In our case DM becomes non-relativistic before the time of matter-radiation equality, $t_{\text {eq }}=1.9 \times 10^{11} \mathrm{~s}$. Thus the free-streaming horizon can be calculated as

$$
\begin{aligned}
r_{\mathrm{FS}} & =\int_{t_{\mathrm{in}}}^{t_{0}} \frac{\langle v(t)\rangle}{a(t)} d t=\int_{t_{\mathrm{in}}}^{t_{\mathrm{nr}}} \frac{1}{a(t)} d t+\int_{t_{\mathrm{nr}}}^{t_{\mathrm{eq}}} \frac{\langle v(t)\rangle}{a(t)} d t+\int_{t_{\mathrm{eq}}}^{t_{0}} \frac{\langle v(t)\rangle}{a(t)} d t \\
& \simeq \frac{2 \sqrt{t_{e q} t_{n r}}}{a_{e q}}+\frac{\sqrt{t_{e q} t_{n r}}}{a_{e q}} \ln \left(\frac{t_{e q}}{t_{n r}}\right)+\frac{3 \sqrt{t_{e q} t_{n r}}}{a_{e q}} \\
& \simeq \frac{\sqrt{t_{e q} t_{n r}}}{a_{e q}}\left[5+\ln \left(\frac{t_{e q}}{t_{n r}}\right)\right] .
\end{aligned}
$$

where $a_{e q}$ is the scale factor at matter-radiation equality. Including entropy dilution the final expression is [26]

$$
r_{\mathrm{FS}} \simeq \frac{\sqrt{t_{e q} t_{n r}}}{a_{e q}}\left[5+\ln \left(\frac{t_{e q}}{t_{n r}}\right)\right] / \xi^{1 / 3} .
$$

where the entropy dilution factor is given by

$$
\xi=\frac{g_{\mathrm{eff}}(\text { highT })}{g_{\mathrm{eff}}\left(\mathrm{t}_{0}\right)} \simeq \frac{109.5}{3.36} .
$$

We have taken into account the scalar field $\sigma$ and the sterile neutrino $N$. DM with a freestreaming scale larger than the size of a dwarf galaxy $\left(r_{F S} \gtrsim 0.1 \mathrm{Mpc}\right)$ constitutes HDM. 


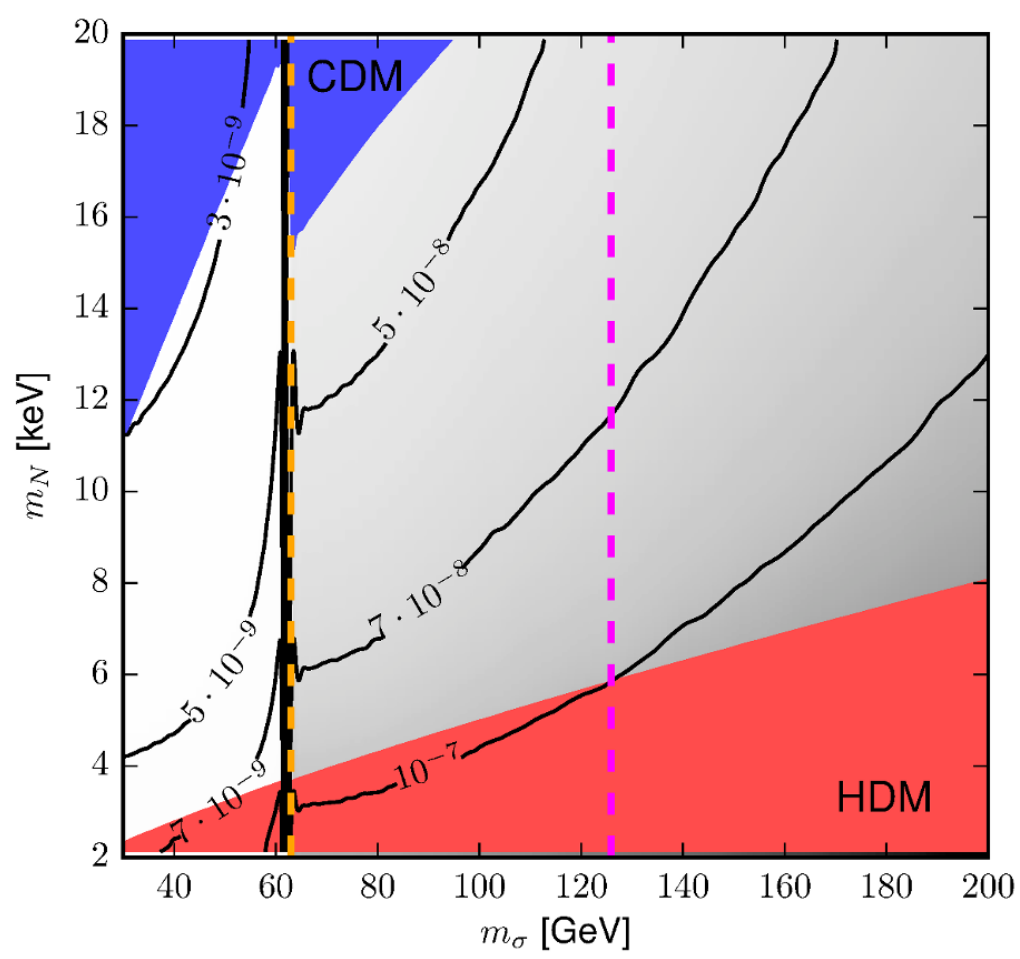

Figure 4. Contour plot of $\lambda_{H \phi}$ in the plane of $m_{N}$ vs $m_{\sigma}$ requiring that the abundance of the keV sterile neutrino can account for the DM abundance at $1 \sigma$, i.e. $0.1172<\Omega_{N} h^{2}<0.1226$ [94]. The (blue) region above $\left(r_{F S} \leq 0.01 \mathrm{Mpc}\right.$.) is the area of CDM while the (red) region below $\left(r_{F S} \geq 0.1\right.$ $\mathrm{Mpc}$ ) is the area of HDM. The area in between $\left(0.01 \mathrm{Mpc} \leq r_{\mathrm{FS}} \leq 0.1 \mathrm{Mpc}\right)$ is the WDM region. The magenta line indicates the Higgs mass $m_{h}$ and the orange line is at $m_{h} / 2$. Below the orange line Higgs decays dominate the production of $\sigma$.

The distinction between WDM and CDM is arbitrary. We follow ref. [26] and consider DM with a free-streaming scale $r_{F S}<0.01 \mathrm{Mpc}$ as CDM and DM with intermediate free-streaming scales as WDM.

The free-streaming horizon depends both on the mass of the scalar field and the mass of sterile neutrino. In figure 3 we plot the free-streaming horizon vs. the keV sterile neutrinos mass for different values of the scalar mass $m_{\sigma}=30,60,65,100,170,500 \mathrm{GeV}$. The HDM (CDM) regions are coloured red (blue). Note that $\mathrm{keV}$ sterile neutrinos with $m_{N}=7.1 \mathrm{keV}$ are WDM for $m_{\sigma} \lesssim 170 \mathrm{GeV}$ and become too hot for larger frozen-in scalar masses. Demanding that the $\mathrm{keV}$ sterile neutrino accounts for the cosmologically observed $\mathrm{DM}$ abundance at $1 \sigma$ and fixing $\lambda_{s}=0.5$, we can plot the required value of the Higgs portal coupling $\lambda_{H \phi}$ as a function of the $\mathrm{keV}$ sterile neutrino mass $m_{N}$ and the frozen-in scalar mass $m_{\sigma}$. This is shown in figure 4 . The blue (red) coloured region indicates the CDM (HDM) region. The black lines are contour lines of equal $\lambda_{H \phi}$ assuming a vanishing activesterile mixing angle, $\theta=0$. The jaggedness of the black contour lines appears because it is not possible to fix the DM abundance to a number, but we only demand it to lie within the $1 \sigma$ allowed region. The grey shading in the background indicates the size of $\lambda_{H \phi}$. Darker 
regions correspond to larger values of $\lambda_{H \phi}$. The magenta line marks the Higgs mass $m_{h}$ and the orange line marks $m_{h} / 2$. To the left of the orange line, Higgs decays will dominate the production of the frozen-in scalar $\sigma$ and the required value of $\lambda_{H \phi}$ is generically smaller. We find $\lambda_{H \phi} \lesssim 10^{-8}$ for $m_{\sigma}<m_{h} / 2$ and $\lambda_{H \phi} \gtrsim 10^{-8}$ for $m_{\sigma}>m_{h} / 2$.

\section{UV completion}

So far we considered an effective operator to generate the neutrino mass. In this section we discuss one simple UV completion using the type-II seesaw mechanism. We introduce a $\mathrm{SU}(2)$ triplet

$$
\Delta=\left(\begin{array}{cc}
\delta^{+} & \delta^{++} \\
v_{\Delta}+\frac{1}{\sqrt{2}}\left(\delta^{0}+i a_{\Delta}\right) & -\delta^{+}
\end{array}\right),
$$

which transforms under $Z_{4}$ as $\Delta \rightarrow-\Delta$. This introduces one more Yukawa coupling

$$
-\mathcal{L}_{\Delta}=\frac{1}{2} y_{L} L^{T}\left(i \sigma^{2} \Delta\right) L+\text { h.c. },
$$

which leads to an additional contribution to neutrino mass after $\Delta$ acquires a vev, and the following additional terms in the scalar potential

$$
\begin{aligned}
V_{\Delta}= & \mu_{\Delta}^{2} \operatorname{Tr}\left(\Delta^{\dagger} \Delta\right)+\frac{\lambda_{\Delta}}{6}\left(\operatorname{Tr}\left(\Delta^{\dagger} \Delta\right)\right)^{2}+\frac{\lambda_{\Delta}^{\prime}}{12} \operatorname{Tr}\left(\left[\Delta^{\dagger}, \Delta\right]^{2}\right) \\
& +\lambda_{H \Delta} H^{\dagger} H \operatorname{Tr}\left(\Delta^{\dagger} \Delta\right)+\lambda_{H \Delta}^{\prime} H^{\dagger}\left[\Delta^{\dagger}, \Delta\right] H+\frac{\lambda_{\phi \Delta}}{2} \phi^{2} \operatorname{Tr}\left(\Delta^{\dagger} \Delta\right) \\
& +\left(\kappa H^{T} i \sigma_{2} \Delta^{\dagger} H \phi+\text { h.c. }\right) .
\end{aligned}
$$

All parameters and vevs can be chosen real by rephasing $H$ and $\Delta$ and there is no spontaneous breaking of CP at tree-level. After $H$ and $\phi$ acquire vevs, a vev is induced for $\Delta$ given by

$$
v_{\Delta}=\kappa \frac{3 \sqrt{6} \mu_{H}^{2} \mu_{\phi}}{\sqrt{\lambda_{\phi}}\left(3 \lambda_{H \Delta} \mu_{H}^{2}+\lambda_{H} \mu_{\Delta}^{2}\right)}
$$

in the limit of a weakly coupled field $\phi$ and a small vev $v_{\Delta}$. There are no charge-breaking minima. The non-vanishing Higgs triplet vev $v_{\Delta}$ will induce a contribution to the $\rho$ parameter. As the scalar $\phi$ and $\mathrm{keV}$ sterile neutrino are very weakly coupled, the constraints from the type-II seesaw model can be directly applied. See ref. [102] for a recent analysis: $v_{\Delta}$ has to be less than $8 \mathrm{GeV}$, which follows from the tree level formula of the electroweak $\rho$ parameter. At leading order there is no mixing between the different scalar fields. The mixing is suppressed by the small Higgs portal couplings, $\lambda_{H \phi}, \lambda_{\Delta \phi}, \kappa$ and the assumption that the vev of $\Delta$ is induced via the coupling $\kappa$.

In the scalar potential (5.3) the only dimensionful coupling is $\mu_{\Delta}$. For scales much smaller than $\mu_{\Delta}$ the type-II seesaw contribution is effectively described by the dimension 6 operator introduced in section 2, i.e. we recover the previously discussed effective theory by integrating out $\Delta$. If the triplet $\Delta$ is heavy enough and its couplings to the scalar $\phi$ 
are sufficiently small, ${ }^{5}$ its contribution to the production of the scalar singlet $\sigma$ via triplet Higgs annihilations $(\Delta \Delta \leftrightarrow \sigma \sigma)$ can be neglected: typical values of $\mu_{\Delta} \gtrsim 10 \mathrm{TeV}$ and $\lambda_{\phi \Delta} / \lambda_{H \phi} \lesssim \mathcal{O}(0.1)$ are sufficient.

Finally we want to comment on possible obstacles to a UV completion using the type-I seesaw mechanism [103-107]. If the right-handed neutrinos carry the same $Z_{4}$ charge, they couple in the same way as the $\mathrm{keV}$ sterile neutrino. As they will be in thermal equilibrium via the Yukawa couplings to the lepton doublets, their couplings to $\phi$ and the keV sterile neutrino $N$ has to be sufficiently suppressed, in particular they are light compared to $v_{\phi}$ loosing the appeal of the type-I seesaw mechanism. However as we noted in section 2, the $Z_{4}$ symmetry is not essential. The DM production mechanism works without the $Z_{4}$ symmetry, but the parameters have to be appropriately tuned to decouple the $\mathrm{keV}$ sterile neutrino and right-handed neutrinos in the usual way. See e.g. refs. [12, 20].

\section{Conclusion}

We studied sterile neutrino dark matter production from the decay of a frozen-in scalar. The previous study of this production mechanism [26] focused on heavy frozen-in scalars with masses above the Higgs mass and light $\mathrm{keV}$ sterile neutrinos with masses below 10 $\mathrm{keV}$ turned out to have a free-streaming scale larger than the size of dwarf galaxies and are thus too hot and excluded.

Motivated by the hints for an X-ray line at $3.55 \mathrm{keV}$, we are considering lighter frozen-in scalars. This leads to a smaller free-streaming horizon and thus lighter sterile neutrinos are allowed. We find that Higgs decay is the dominant production channel when kinematically allowed. For $m_{\sigma}>m_{h} / 2$ Higgs decay becomes kinematically forbidden and the main production channels are annihilation into Higgs pairs as well as pairs of EW gauge bosons, particularly $W$-bosons. Above the EW phase transition the frozen-in scalar and thus the $\mathrm{keV}$ sterile neutrino is only produced by Higgs annihilations.

Furthermore we calculated the free-streaming horizon to show the viable region in parameter space, where sterile neutrinos are WDM or CDM. A $7.1 \mathrm{keV}$ sterile neutrino requires the frozen-in scalar to be lighter than approximately $170 \mathrm{GeV}$. Demanding that the keV sterile neutrino accounts for the cosmological DM abundance, we determined the necessary value of the Higgs portal coupling $\lambda_{H \phi}$. Above $m_{h} / 2$ the coupling is $\lambda_{H \phi} \gtrsim 10^{-8}$, below $m_{h} / 2$ the coupling is generically smaller, $\lambda_{H \phi} \lesssim 10^{-8}$.

Although we studied the DM production in an effective theory for neutrino mass, we showed a simple UV completion using the type-II seesaw mechanism [82-87] in section 5 . As long as the triplet is heavy enough and its couplings to the scalar $\phi$ are small enough, it can be neglected in the discussion of the production mechanism.

We discussed a minimal model of a $\mathrm{keV}$ sterile neutrino production from a frozenin scalar. It might be interesting to promote the discrete $Z_{4}$ symmetry to a continuous $\mathrm{U}(1)$. This introduces a (pseudo) Goldstone boson (pGB) which substantially modifies the

\footnotetext{
${ }^{5}$ All quantum corrections to these couplings are either proportional to the coupling itself or the coupling $\lambda_{H \phi}$. Hence it is safe to assume that the couplings are sufficiently suppressed compared to $\lambda_{H \phi}$, such that they can be neglected.
} 
production. As we expect the pGB to have strong couplings to the scalar $\sigma$ via its quartic interaction, it will be efficiently produced and the cosmological DM abundance might be explained by a mixture of $\mathrm{keV}$ sterile neutrinos and the pGB.

\section{Acknowledgments}

We would like to thank Alexander Merle and Viviana Niro for carefully reading our manuscript and their valuable comments. AA would like to thank Piyabut Burikham for his useful advice. This work has been supported by the Australian Research Council and Chulalongkorn University through Ratchadapisek Sompote Endowment Fund.

\section{A Cross sections and decay widths}

The relevant cross sections $W_{a b}=4 E_{a} E_{b} \sigma v$ for annihilation into fermions $W_{f f}$, vector bosons $W_{V V}$ and the Higgs $W_{h h}{ }^{6}$ are given by [108]

$$
\begin{aligned}
W_{h h} & =\frac{\lambda_{H \phi}^{2}}{16 \pi} \sqrt{1-\frac{4 m_{h}^{2}}{s}}\left(\frac{s+2 m_{h}^{2}}{s-m_{h}^{2}}\right)^{2} \\
W_{t \bar{t}} & =\frac{\lambda_{H \phi}^{2} m_{t}^{2}}{4 \pi} \frac{1}{\left(s-m_{h}^{2}\right)^{2}+m_{h}^{2} \Gamma_{h}^{2}} \frac{\left(s-4 m_{t}^{2}\right)^{3 / 2}}{\sqrt{s}} \\
W_{V V} & =\frac{\lambda_{H \phi}^{2}}{16 \pi} \frac{1}{\left(s-m_{h}^{2}\right)^{2}+m_{h}^{2} \Gamma_{h}^{2}} \sqrt{1-\frac{4 m_{V}^{2}}{s}}\left(1-\frac{4 m_{V}^{2}}{s}+\frac{12 m_{V}^{4}}{s^{2}}\right) .
\end{aligned}
$$

The annihilation into $Z(W)$-bosons is

$$
W_{Z Z}=W_{V V}\left(m_{V}=m_{Z}\right) \quad W_{W W}=2 W_{V V}\left(m_{V}=m_{W}\right) .
$$

The partial Higgs decay width to scalars is given by

$$
\Gamma(h \rightarrow \sigma \sigma)=\frac{\left|\lambda_{H \phi}\right|^{2} v^{2}}{16 \pi m_{h}}\left(1-\frac{4 m_{\sigma}^{2}}{m_{h}^{2}}\right)^{1 / 2}
$$

and the partial decay widths of $\sigma$ as well as the Higgs to two keV neutrinos $N$ are described by

$$
\begin{aligned}
& \Gamma(\sigma \rightarrow N N)=\frac{y_{N}^{2}}{16 \pi} m_{\phi}\left(1-\frac{4 m_{N}^{2}}{m_{\sigma}^{2}}\right)^{3 / 2} \\
& \Gamma(h \rightarrow N N)=\frac{y_{N}^{2} \sin ^{2} \gamma}{16 \pi} m_{h}\left(1-\frac{4 m_{N}^{2}}{m_{h}^{2}}\right)^{3 / 2}
\end{aligned}
$$

where $y_{N}$ is chosen to be real without loss of generality and $\sin \gamma$ denotes the mixing between the Higgs and the scalar $\sigma$, which is defined in eq. (2.7).

\footnotetext{
${ }^{6}$ For simplicity we approximated the Higgs annihilation cross section and neglect higher order terms in $\lambda_{H \phi}$. They are shown in ref. [108].
} 


\section{B Thermal averages}

The thermal average of a partial decay width $\Gamma(X \rightarrow i i)$ of a particle $X$ with mass $m_{X}$ can be calculated as follows

$$
\langle\Gamma(X \rightarrow i i)\rangle=\Gamma(X \rightarrow i i) \frac{K_{1}(x)}{K_{2}(x)}
$$

with $x=m_{X} / T$. Following refs. [108, 109], we write the thermally averaged annihilation cross section, $\langle\sigma v\rangle$, as

$$
\langle\sigma v(\sigma \sigma \rightarrow a b)\rangle=g_{a} g_{b} \frac{m_{\sigma}}{64 \pi^{4} x n_{e q}^{2}} \int_{4 m_{\sigma}^{2}}^{\infty} W_{a b} \sqrt{1-\frac{4 m_{\sigma}^{2}}{s}} \sqrt{s} K_{1}\left(\frac{x \sqrt{s}}{m_{\sigma}}\right) \mathrm{d} s
$$

where $x=m_{\sigma} / T$ and $n_{e q}$ denotes the equilibrium number density of $\sigma$. It is given by

$$
n_{e q}=\frac{g_{\sigma}}{2 \pi^{2}} \frac{m_{\sigma}^{3}}{x} K_{2}(x)
$$

where $g_{\sigma}=2 s_{\sigma}+1$ are the spin degrees of freedom of $\sigma$ and $K_{2}(x)$ denotes the modified Bessel function of second kind. $W_{a b}$ is defined as $4 E_{a} E_{b} \sigma v$. The relevant cross sections $W_{a b}$ are given in appendix A.

\section{Effective degree of freedom}

We follow the discussion in [110]. The energy density and the entropy density are defined as

$$
\rho(T)=g_{\mathrm{eff}}(T) \frac{\pi^{2}}{30} T^{4} \quad s(T)=h_{\mathrm{eff}}(T) \frac{2 \pi^{2}}{45} T^{3} .
$$

In order to define the total effective number of degrees of freedom, we first have to define the effective number of degrees of freedom for energy and entropy for each individual particle species $i$

$$
\begin{aligned}
& g_{i}(T)=\frac{30}{\pi^{2} T^{4}} \rho_{i}(T)=\frac{15 g_{i}}{\pi^{4}} x_{i}^{4} \int_{1}^{\infty} \frac{y\left(y^{2}-1\right)^{1 / 2}}{e^{x_{i} y}+\eta_{i}} y d y \\
& h_{i}(T)=\frac{45}{2 \pi^{2} T^{3}} s_{i}(T)=\frac{45 g_{i}}{4 \pi^{4}} x_{i}^{4} \int_{1}^{\infty} \frac{y\left(y^{2}-1\right)^{1 / 2}}{e^{x_{i} y}+\eta_{i}} \frac{4 y^{2}-1}{3 y} d y,
\end{aligned}
$$

where $\rho_{i}(T)\left(s_{i}(T)\right)$ is the energy (entropy) density of each particle species and $x_{i}=m_{i} / T$ with $m_{i}$ being the mass of the particle species.

The total energy effective degrees of freedom is

$$
g_{\mathrm{eff}}(T)=\sum_{T_{d_{i}}<T} g_{i}(T)+\sum_{i \in \mathrm{dec}} g_{i}(T) \frac{T_{i}^{4}}{T^{4}} \equiv g_{c}(T)+\sum_{i \in \operatorname{dec}} g_{i}(T) \frac{T_{i}^{4}}{T^{4}},
$$

where $g_{c}(T)$ is the energy effective degree of freedom including all coupled species at temperature $T$. The second contribution includes all species that are already decoupled at temperature $T$. Like in [110], we also choose $T_{d_{i}}=m_{i} / 20$ for the decoupling temperature. 
The total entropy effective degrees of freedom can be calculated as

$$
h_{\mathrm{eff}}(T)=h_{c}(T) \prod_{i \in \mathrm{dec}}\left(1+\frac{h_{i}\left(T_{d_{i}}\right)}{h_{c}\left(T_{d_{i}}\right)}\right),
$$

where $h_{c}(T)=\sum_{T_{d_{i}}<T} h_{i}(T)$ is the entropy effective degree of freedom including all coupled species at temperature $T$ and the product here extends to all the species that are already decoupled at temperature $T$. The effective degrees of freedom parameter $g_{*}^{1 / 2}$ is defined following refs. $[26,110]$ as

$$
\sqrt{g_{*}(T)}=\frac{h_{\mathrm{eff}}(T)}{\sqrt{g_{\mathrm{eff}}(T)}}\left(1+\frac{1}{3} \frac{T}{h_{\mathrm{eff}}(T)} \frac{\mathrm{d} h_{\mathrm{eff}}(T)}{\mathrm{d} T}\right) .
$$

Open Access. This article is distributed under the terms of the Creative Commons Attribution License (CC-BY 4.0), which permits any use, distribution and reproduction in any medium, provided the original author(s) and source are credited.

\section{References}

[1] K.N. Abazajian et al., Light sterile neutrinos: a white paper, arXiv:1204.5379 [INSPIRE].

[2] A. Kusenko, F. Takahashi and T.T. Yanagida, Dark matter from split seesaw, Phys. Lett. B 693 (2010) 144 [arXiv: 1006.1731] [INSPIRE].

[3] M. Lindner, A. Merle and V. Niro, Soft $L_{e}-L_{\mu}-L_{\tau}$ flavour symmetry breaking and sterile neutrino keV dark matter, JCAP 01 (2011) 034 [Erratum ibid. 07 (2014) E01] [arXiv:1011.4950] [INSPIRE].

[4] A. Adulpravitchai and R. Takahashi, $A_{4}$ flavor models in split seesaw mechanism, JHEP 09 (2011) 127 [arXiv: 1107.3829] [inSPIRE].

[5] A. Merle and V. Niro, Deriving models for keV sterile neutrino dark matter with the Froggatt-Nielsen mechanism, JCAP 07 (2011) 023 [arXiv:1105.5136] [INSPIRE].

[6] J. Barry, W. Rodejohann and H. Zhang, Sterile neutrinos for warm dark matter and the reactor anomaly in flavor symmetry models, JCAP 01 (2012) 052 [arXiv:1110.6382] [INSPIRE].

[7] A. Merle, keV neutrino model building, Int. J. Mod. Phys. D 22 (2013) 1330020 [arXiv:1302.2625] [INSPIRE].

[8] R. Takahashi, Separate seesaw and its applications to dark matter and baryogenesis, Prog. Theor. Exp. Phys. 2013 (2013) 063B04 [arXiv: 1303. 0108] [INSPIRE].

[9] G. Kauffmann, S.D.M. White and B. Guiderdoni, The formation and evolution of galaxies within merging dark matter haloes, Mon. Not. Roy. Astron. Soc. 264 (1993) 201 [INSPIRE].

[10] A.A. Klypin, A.V. Kravtsov, O. Valenzuela and F. Prada, Where are the missing galactic satellites?, Astrophys. J. $\mathbf{5 2 2}$ (1999) 82 [astro-ph/9901240] [INSPIRE].

[11] B. Moore, J. Diemand, P. Madau, M. Zemp and J. Stadel, Globular clusters, satellite galaxies and stellar haloes from early dark matter peaks, Mon. Not. Roy. Astron. Soc. 368 (2006) 563 [astro-ph/0510370] [INSPIRE]. 
[12] A. Kusenko, Sterile neutrinos, dark matter and the pulsar velocities in models with a Higgs singlet, Phys. Rev. Lett. 97 (2006) 241301 [hep-ph/0609081] [INSPIRE].

[13] A. Kusenko and G. Segre, Neutral current induced neutrino oscillations in a supernova, Phys. Lett. B 396 (1997) 197 [hep-ph/9701311] [INSPIRE].

[14] R. Barbieri and A. Dolgov, Neutrino oscillations in the early universe, Nucl. Phys. B 349 (1991) 743 [INSPIRE].

[15] K. Enqvist, K. Kainulainen and J. Maalampi, Refraction and oscillations of neutrinos in the early universe, Nucl. Phys. B 349 (1991) 754 [INSPIRE].

[16] S. Dodelson and L.M. Widrow, Sterile-neutrinos as dark matter, Phys. Rev. Lett. 72 (1994) 17 [hep-ph/9303287] [INSPIRE].

[17] L. Canetti, M. Drewes, T. Frossard and M. Shaposhnikov, Dark matter, baryogenesis and neutrino oscillations from right handed neutrinos, Phys. Rev. D 87 (2013) 093006 [arXiv: 1208.4607] [INSPIRE].

[18] L. Canetti, M. Drewes and M. Shaposhnikov, Sterile neutrinos as the origin of dark and baryonic matter, Phys. Rev. Lett. 110 (2013) 061801 [arXiv: 1204.3902] [INSPIRE].

[19] X.-D. Shi and G.M. Fuller, A new dark matter candidate: nonthermal sterile neutrinos, Phys. Rev. Lett. 82 (1999) 2832 [astro-ph/9810076] [INSPIRE].

[20] K. Petraki and A. Kusenko, Dark-matter sterile neutrinos in models with a gauge singlet in the Higgs sector, Phys. Rev. D 77 (2008) 065014 [arXiv:0711.4646] [INSPIRE].

[21] M. Frigerio and C.E. Yaguna, Sterile neutrino dark matter and low scale leptogenesis from a charged scalar, arXiv:1409.0659 [INSPIRE].

[22] M. Shaposhnikov and I. Tkachev, The $\nu M S M$, inflation and dark matter, Phys. Lett. B 639 (2006) 414 [hep-ph/0604236] [INSPIRE].

[23] F. Bezrukov and D. Gorbunov, Light inflaton hunter's guide, JHEP 05 (2010) 010 [arXiv:0912.0390] [INSPIRE].

[24] F. Bezrukov, H. Hettmansperger and M. Lindner, keV sterile neutrino dark matter in gauge extensions of the standard model, Phys. Rev. D 81 (2010) 085032 [arXiv:0912.4415] [INSPIRE].

[25] M. Nemevšek, G. Senjanović and Y. Zhang, Warm dark matter in low scale left-right theory, JCAP 07 (2012) 006 [arXiv:1205.0844] [INSPIRE].

[26] A. Merle, V. Niro and D. Schmidt, New production mechanism for keV sterile neutrino dark matter by decays of frozen-in scalars, JCAP 03 (2014) 028 [arXiv: 1306.3996] [INSPIRE].

[27] A. Aparici, A. Santamaria and J. Wudka, A model for right-handed neutrino magnetic moments, J. Phys. G 37 (2010) 075012 [arXiv:0911.4103] [INSPIRE].

[28] E. Bulbul et al., Detection of an unidentified emission line in the stacked X-ray spectrum of galaxy clusters, Astrophys. J. 789 (2014) 13 [arXiv:1402.2301] [INSPIRE].

[29] A. Boyarsky, O. Ruchayskiy, D. Iakubovskyi and J. Franse, An unidentified line in X-ray spectra of the Andromeda galaxy and Perseus galaxy cluster, Phys. Rev. Lett. 113 (2014) 251301 [arXiv: 1402.4119] [INSPIRE].

[30] H. Ishida, K.S. Jeong and F. Takahashi, $7 \mathrm{keV}$ sterile neutrino dark matter from split flavor mechanism, Phys. Lett. B 732 (2014) 196 [arXiv:1402.5837] [INSPIRE]. 
[31] K.N. Abazajian, Resonantly produced $7 \mathrm{keV}$ sterile neutrino dark matter models and the properties of Milky Way satellites, Phys. Rev. Lett. 112 (2014) 161303 [arXiv:1403.0954] [INSPIRE].

[32] F. Bezrukov and D. Gorbunov, Relic gravity waves and 7 keV dark matter from a GeV scale inflaton, Phys. Lett. B 736 (2014) 494 [arXiv:1403.4638] [INSPIRE].

[33] S. Chakraborty, D.K. Ghosh and S. Roy, 7 keV sterile neutrino dark matter in $\mathrm{U}(1)_{R}$-lepton number model, JHEP 10 (2014) 146 [arXiv:1405.6967] [INSPIRE].

[34] N. Haba, H. Ishida and R. Takahashi, $\nu_{R}$ dark matter-philic Higgs for 3.5 keV X-ray signal, arXiv:1407.6827 [INSPIRE].

[35] D.J. Robinson and Y. Tsai, Dynamical framework for KeV Dirac neutrino warm dark matter, Phys. Rev. D 90 (2014) 045030 [arXiv:1404.7118] [INSPIRE].

[36] W. Rodejohann and H. Zhang, Signatures of extra dimensional sterile neutrinos, Phys. Lett. B 737 (2014) 81 [arXiv:1407.2739] [INSPIRE].

[37] S. Patra and P. Pritimita, 7 keV sterile neutrino dark matter in extended seesaw framework, arXiv:1409.3656 [INSPIRE].

[38] T. Higaki, K.S. Jeong and F. Takahashi, The 7 keV axion dark matter and the X-ray line signal, Phys. Lett. B 733 (2014) 25 [arXiv:1402.6965] [INSPIRE].

[39] J.-C. Park, S.C. Park and K. Kong, X-ray line signal from 7 keV axino dark matter decay, Phys. Lett. B 733 (2014) 217 [arXiv:1403.1536] [InSPIRE].

[40] M.T. Frandsen, F. Sannino, I.M. Shoemaker and O. Svendsen, X-ray lines from dark matter: the good, the bad and the unlikely, JCAP 05 (2014) 033 [arXiv:1403.1570] [InSPIRE].

[41] S. Baek and H. Okada, 7 keV dark matter as X-ray line signal in radiative neutrino model, arXiv: 1403.1710 [INSPIRE].

[42] K.S. Babu and R.N. Mohapatra, $7 \mathrm{keV}$ scalar dark matter and the anomalous galactic X-ray spectrum, Phys. Rev. D 89 (2014) 115011 [arXiv:1404.2220] [INSPIRE].

[43] C. Kolda and J. Unwin, X-ray lines from R-parity violating decays of ke $V$ sparticles, Phys. Rev. D 90 (2014) 023535 [arXiv: 1403.5580] [INSPIRE].

[44] F.S. Queiroz and K. Sinha, The poker face of the Majoron dark matter model: LUX to keV line, Phys. Lett. B 735 (2014) 69 [arXiv:1404.1400] [INSPIRE].

[45] K.-Y. Choi and O. Seto, X-ray line signal from decaying axino warm dark matter, Phys. Lett. B 735 (2014) 92 [arXiv:1403.1782] [InSPIRE].

[46] S.P. Liew, Axino dark matter in light of an anomalous X-ray line, JCAP 05 (2014) 044 [arXiv: 1403.6621] [INSPIRE].

[47] Z. Kang, P. Ko, T. Li and Y. Liu, Natural X-ray lines from the low scale supersymmetry breaking, arXiv:1403.7742 [INSPIRE].

[48] E. Dudas, L. Heurtier and Y. Mambrini, Generating X-ray lines from annihilating dark matter, Phys. Rev. D 90 (2014) 035002 [arXiv:1404.1927] [INSPIRE].

[49] C.-Q. Geng, D. Huang and L.-H. Tsai, X-ray line from the dark transition electric dipole, JHEP 08 (2014) 086 [arXiv:1406.6481] [INSPIRE].

[50] C.-W. Chiang and T. Yamada, $3.5 \mathrm{keV}$ X-ray line from nearly-degenerate WIMP dark matter decays, JHEP 09 (2014) 006 [arXiv: 1407.0460] [INSPIRE]. 
[51] J. Jaeckel, J. Redondo and A. Ringwald, $3.55 \mathrm{keV}$ hint for decaying axionlike particle dark matter, Phys. Rev. D 89 (2014) 103511 [arXiv:1402.7335] [INSPIRE].

[52] M. Cicoli, J.P. Conlon, M.C.D. Marsh and M. Rummel, $3.55 \mathrm{keV}$ photon line and its morphology from a $3.55 \mathrm{keV}$ axionlike particle line, Phys. Rev. D 90 (2014) 023540 [arXiv:1403.2370] [INSPIRE].

[53] H. Okada and T. Toma, $3.55 \mathrm{keV} \mathrm{X-ray} \mathrm{line} \mathrm{signal} \mathrm{from} \mathrm{excited} \mathrm{dark} \mathrm{matter} \mathrm{in} \mathrm{radiative}$ neutrino model, Phys. Lett. B 737 (2014) 162 [arXiv:1404.4795] [InSPIRE].

[54] H.M. Lee, Magnetic dark matter for the X-ray line at 3.55 keV, Phys. Lett. B 738 (2014) 118 [arXiv: 1404.5446] [INSPIRE].

[55] J.P. Conlon and F.V. Day, $3.55 \mathrm{keV}$ photon lines from axion to photon conversion in the Milky Way and M31, arXiv:1404.7741 [INSPIRE].

[56] J.P. Conlon and A.J. Powell, A $3.55 \mathrm{keV}$ line from $D M \rightarrow a \rightarrow \gamma$ : predictions for cool-core and non-cool-core clusters, arXiv: 1406.5518 [INSPIRE].

[57] H. Ishida and H. Okada, $3.55 \mathrm{keV} X$-ray line interpretation in radiative neutrino model, arXiv: 1406.5808 [INSPIRE].

[58] V.K. Dubrovich, Identification of $3.55 \mathrm{keV}$ line in the framework of standard physics, arXiv: 1407.4629 [INSPIRE].

[59] K. Nakayama, F. Takahashi and T.T. Yanagida, The $3.5 \mathrm{keV}$ X-ray line signal from decaying moduli with low cutoff scale, Phys. Lett. B 735 (2014) 338 [arXiv:1403.1733] [INSPIRE].

[60] N.E. Bomark and L. Roszkowski, $3.5 \mathrm{keV} \mathrm{X-ray} \mathrm{line} \mathrm{from} \mathrm{decaying} \mathrm{gravitino} \mathrm{dark} \mathrm{matter,}$ Phys. Rev. D 90 (2014) 011701 [arXiv:1403.6503] [INSPIRE].

[61] K. Nakayama, F. Takahashi and T.T. Yanagida, Anomaly-free flavor models for Nambu-Goldstone bosons and the $3.5 \mathrm{keV} \mathrm{X-ray} \mathrm{line} \mathrm{signal,} \mathrm{Phys.} \mathrm{Lett.} \mathrm{B} 734$ (2014) 178 [arXiv:1403.7390] [INSPIRE].

[62] K.P. Modak, $3.5 \mathrm{keV}$ X-ray line signal from decay of right-handed neutrino due to transition magnetic moment, arXiv: 1404.3676 [INSPIRE].

[63] J.M. Cline, Y. Farzan, Z. Liu, G.D. Moore and W. Xue, $3.5 \mathrm{keV}$ X-rays as the "21 cm line" of dark atoms and a link to light sterile neutrinos, Phys. Rev. D 89 (2014) 121302 [arXiv: 1404.3729] [INSPIRE].

[64] S. Baek, P. Ko and W.-I. Park, The $3.5 \mathrm{keV} \mathrm{X-ray} \mathrm{line} \mathrm{signature} \mathrm{from} \mathrm{annihilating} \mathrm{and}$ decaying dark matter in Weinberg model, arXiv:1405.3730 [INSPIRE].

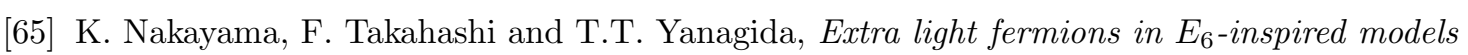
and the $3.5 \mathrm{keV}$ X-ray line signal, Phys. Lett. B 737 (2014) 311 [arXiv:1405.4670] [INSPIRE].

[66] N. Chen, Z. Liu and P. Nath, $3.5 \mathrm{keV}$ galactic emission line as a signal from the hidden sector, Phys. Rev. D 90 (2014) 035009 [arXiv: 1406.0687] [INSPIRE].

[67] B. Dutta, I. Gogoladze, R. Khalid and Q. Shafi, $3.5 \mathrm{keV} X$-ray line and R-parity conserving supersymmetry, JHEP 11 (2014) 018 [arXiv:1407.0863] [INSPIRE].

[68] J.M. Cline and A.R. Frey, Non-Abelian dark matter models for $3.5 \mathrm{keV}$ X-rays, JCAP 10 (2014) 013 [arXiv: 1408.0233] [INSPIRE].

[69] T. Higaki, N. Kitajima and F. Takahashi, Hidden axion dark matter decaying through mixing with $Q C D$ axion and the $3.5 \mathrm{keV} X$-ray line, arXiv:1408.3936 [INSPIRE]. 
[70] Y. Farzan and A.R. Akbarieh, Decaying vector dark matter as an explanation for the $3.5 \mathrm{keV}$ line from galaxy clusters, JCAP 11 (2014) 015 [arXiv: 1408.2950] [INSPIRE].

[71] H. Okada and Y. Orikasa, X-ray line in radiative neutrino model with global U(1) symmetry, Phys. Rev. D 90 (2014) 075023 [arXiv:1407.2543] [INSPIRE].

[72] G. Faisel, S.-Y. Ho and J. Tandean, Exploring X-ray lines as scotogenic signals, Phys. Lett. B 738 (2014) 380 [arXiv: 1408.5887] [INSPIRE].

[73] K.K. Boddy, J.L. Feng, M. Kaplinghat, Y. Shadmi and T.M.P. Tait, Strongly interacting dark matter: self-interactions and keV lines, Phys. Rev. D 90 (2014) 095016 [arXiv: 1408.6532] [INSPIRE].

[74] K. Schutz and T.R. Slatyer, Self-scattering for dark matter with an excited state, arXiv: 1409.2867 [INSPIRE].

[75] A. Falkowski, Y. Hochberg and J.T. Ruderman, Displaced vertices from X-ray lines, JHEP 11 (2014) 140 [arXiv: 1409.2872] [INSPIRE].

[76] A. Boyarsky, J. Franse, D. Iakubovskyi and O. Ruchayskiy, Checking the dark matter origin of $3.53 \mathrm{keV}$ line with the Milky Way center, arXiv:1408.2503 [INSPIRE].

[77] A. Boyarsky, J. Franse, D. Iakubovskyi and O. Ruchayskiy, Comment on the paper "dark matter searches going bananas: the contribution of Potassium (and Chlorine) to the $3.5 \mathrm{keV}$ line" by T. Jeltema and S. Profumo, arXiv:1408.4388 [inSPIRE].

[78] S. Riemer-Sorensen, Questioning a $3.5 \mathrm{keV}$ dark matter emission line, arXiv: 1405.7943 [INSPIRE].

[79] T.E. Jeltema and S. Profumo, Dark matter searches going bananas: the contribution of Potassium (and Chlorine) to the $3.5 \mathrm{keV}$ line, arXiv:1408.1699 [INSPIRE].

[80] D. Malyshev, A. Neronov and D. Eckert, Constraints on $3.55 \mathrm{keV}$ line emission from stacked observations of dwarf spheroidal galaxies, Phys. Rev. D 90 (2014) 103506 [arXiv: 1408.3531] [INSPIRE].

[81] K. Enqvist, S. Nurmi, T. Tenkanen and K. Tuominen, Standard model with a real singlet scalar and inflation, JCAP 08 (2014) 035 [arXiv: 1407.0659] [INSPIRE].

[82] M. Magg and C. Wetterich, Neutrino mass problem and gauge hierarchy, Phys. Lett. B 94 (1980) 61 [INSPIRE].

[83] J. Schechter and J.W.F. Valle, Neutrino masses in $\mathrm{SU}(2) \times \mathrm{U}(1)$ theories, Phys. Rev. D 22 (1980) 2227 [INSPIRE].

[84] C. Wetterich, Neutrino masses and the scale of B-L violation, Nucl. Phys. B 187 (1981) 343 [INSPIRE].

[85] G. Lazarides, Q. Shafi and C. Wetterich, Proton lifetime and fermion masses in an $\mathrm{SO}(10)$ model, Nucl. Phys. B 181 (1981) 287 [inSPIRE].

[86] R.N. Mohapatra and G. Senjanović, Neutrino masses and mixings in gauge models with spontaneous parity violation, Phys. Rev. D 23 (1981) 165 [INSPIRE].

[87] T.P. Cheng and L.-F. Li, Neutrino masses, mixings and oscillations in $\mathrm{SU}(2) \times \mathrm{U}(1)$ models of electroweak interactions, Phys. Rev. D 22 (1980) 2860 [INSPIRE].

[88] Y. Zeldovich, I.Y. Kobzarev and L.B. Okun, Cosmological consequences of the spontaneous breakdown of discrete symmetry, Zh. Eksp. Teor. Fiz. 67 (1974) 3 [Sov. Phys. JETP 40 (1974) 1] [INSPIRE]. 
[89] F. Riva, Low-scale leptogenesis and the domain wall problem in models with discrete flavor symmetries, Phys. Lett. B 690 (2010) 443 [arXiv: 1004.1177] [INSPIRE].

[90] S.E. Larsson, S. Sarkar and P.L. White, Evading the cosmological domain wall problem, Phys. Rev. D 55 (1997) 5129 [hep-ph/9608319] [INSPIRE].

[91] G.R. Dvali, A. Melfo and G. Senjanović, Nonrestoration of spontaneously broken P and CP at high temperature, Phys. Rev. D 54 (1996) 7857 [hep-ph/9601376] [INSPIRE].

[92] G.R. Dvali and G. Senjanović, Is there a domain wall problem?, Phys. Rev. Lett. 74 (1995) 5178 [hep-ph/9501387] [INSPIRE].

[93] J. Preskill, S.P. Trivedi, F. Wilczek and M.B. Wise, Cosmology and broken discrete symmetry, Nucl. Phys. B 363 (1991) 207 [INSPIRE].

[94] Planck collaboration, P.A.R. Ade et al., Planck 2013 results. XVI. Cosmological parameters, Astron. Astrophys. 571 (2014) A16 [arXiv:1303.5076] [INSPIRE].

[95] A. Kusenko, Sterile neutrinos: the dark side of the light fermions, Phys. Rept. 481 (2009) 1 [arXiv:0906.2968] [INSPIRE].

[96] M. Frigerio, T. Hambye and E. Masso, Sub-GeV dark matter as pseudo-Goldstone from the seesaw scale, Phys. Rev. X 1 (2011) 021026 [arXiv:1107.4564] [InSPIRE].

[97] J. Hasenkamp and J. Kersten, Dark radiation from particle decay: cosmological constraints and opportunities, JCAP 08 (2013) 024 [arXiv:1212.4160] [INSPIRE].

[98] A. Kamada, N. Yoshida, K. Kohri and T. Takahashi, Structure of dark matter halos in warm dark matter models and in models with long-lived charged massive particles, JCAP 03 (2013) 008 [arXiv: 1301.2744] [InSPIRE].

[99] S. Aoyama, K. Ichiki, D. Nitta and N. Sugiyama, Formulation and constraints on decaying dark matter with finite mass daughter particles, JCAP 09 (2011) 025 [arXiv:1106.1984] [INSPIRE].

[100] L.E. Strigari, M. Kaplinghat and J.S. Bullock, Dark matter halos with cores from hierarchical structure formation, Phys. Rev. D 75 (2007) 061303 [astro-ph/0606281] [INSPIRE].

[101] M. Kaplinghat, Dark matter from early decays, Phys. Rev. D 72 (2005) 063510 [astro-ph/0507300] [INSPIRE].

[102] M. Aoki, S. Kanemura, M. Kikuchi and K. Yagyu, Radiative corrections to the Higgs boson couplings in the triplet model, Phys. Rev. D 87 (2013) 015012 [arXiv:1211.6029] [INSPIRE].

[103] P. Minkowski, $\mu \rightarrow$ er at a rate of one out of 1-billion muon decays?, Phys. Lett. B 67 (1977) 421 [INSPIRE].

[104] T. Yanagida, Horizontal symmetry and masses of neutrinos, in Proceedings of the Workshop on The Unified Theory and the Baryon Number in the Universe, O. Sawada and

A. Sugamoto eds., KEK, Tsukuba Japan (1979), pg. 79 [INSPIRE].

[105] S.L. Glashow, The future of elementary particle physics, in Proceedings of the 1979 Cargèse Summer Institute on Quarks and Leptons, M. Lévy et al. eds., Plenum Press, New York U.S.A. (1980), pg. 687 [INSPIRE].

[106] M. Gell-Mann, P. Ramond and R. Slansky, Complex spinors and unified theories, in Supergravity, P. van Nieuwenhuizen and D.Z. Freedman eds., North Holland, Amsterdam The Netherlands (1979), pg. 315 [arXiv:1306.4669] [INSPIRE]. 
[107] R.N. Mohapatra and G. Senjanović, Neutrino mass and spontaneous parity violation, Phys. Rev. Lett. 44 (1980) 912 [INSPIRE].

[108] W.-L. Guo and Y.-L. Wu, The real singlet scalar dark matter model, JHEP 10 (2010) 083 [arXiv: 1006.2518] [INSPIRE].

[109] A. Adulpravitchai, B. Batell and J. Pradler, Non-Abelian discrete dark matter, Phys. Lett. B 700 (2011) 207 [arXiv: 1103.3053] [INSPIRE].

[110] P. Gondolo and G. Gelmini, Cosmic abundances of stable particles: improved analysis, Nucl. Phys. B 360 (1991) 145 [INSPIRE]. 